\title{
Efficiency and Learnability Comparison of the Gesture-based and the Mouse-based Telerobotic Systems
}

\author{
Jinyi YU ${ }^{1}$, Woojin PAIK ${ }^{2 *}$ \\ ${ }^{1}$ School of Science, Central South University of Forestry and Technology, Changsha 410004, China \\ wujinyi994@gmail.com \\ ${ }^{2}$ Dept. of Software Technology, Konkuk University Glocal Campus, Chungju 27478, Republic of Korea \\ wjpaik@kku.ac.kr (*Corresponding author)
}

\begin{abstract}
Telerobotic systems enable operators to interact with remote manipulators. Such systems have often been used in hazardous environments. This paper presents a gesture-based telerobotic system using Kinect. Kinect is an essential component of the Natural User Interface (NUI) capable of translating human body motion into telemanipulation commands. In comparison to traditional mouse-based control, the gesture-based system led to faster completion of the remote object moving task and was also easier to learn. Thus, it is possible to conclude that the gesture-based NUI proved a more intuitive and efficient usage than the traditional user interfaces.
\end{abstract}

Keywords: Telerobotic, Gesture-based control, Natural User Interface, Kinect

\section{Introduction}

Robotics include telerobotics, which involves controlling semi-autonomous robots from a distance using communication networks such as Wi-Fi, Bluetooth, and the Deep Space Network. The aim of a telerobotic system is to link humans and robots so as to reproduce the human controller's operation in the robot movements at a distance. Vision and control are the two primary functions of telerobotics and telepresence. The remote cameras provide the human operators with an intuitive and immersive control experience.

There is a varying degree of fidelity in the different telerobotic interfaces. The most common interface is Monitor-Mouse-Keyboard (MMK), which is inexpensive to develop and not immersive. Another type of interfaces uses joysticks, which provides intuitive 2D planar manipulation experiences. The other type utilizes a Head Mounted Display (HMD) with either single or binocular displays. All three telerobotic interfaces types are difficult to use, as the controls do not easily comply with the manner in which the telerobots move and often lead to 'simulator sickness', especially in case of the HMD type. In summary, one of the main obstacles to the widespread use of telerobotics is represented by the complicated manipulations.

This study presents a gesture-based telerobotic system using Kinect. Kinect is an essential component of the alternative Natural User Interface (NUI) that is capable of translating human body motion into telemanipulation commands. The experiments were conducted to determine how quickly telerobotic system users can adapt to the new interface and also to compare how quickly they can complete the given simple task between the traditional and the proposed gesture-based interface setups. The primary hypothesis of this study was that the gesturebased telerobotic operation would be faster to command than the baseline mouse-based control. The secondary hypothesis was that it would be easier to learn to use the gesture-based interface than the mouse-based system.

Section 2 presents the literature review to situate the presented research. Section 3 describes how the robot arm was constructed for the experiment and the programming aspect of manipulating the robot arm in section 4. Section 5 shows three experimental setups and the experiment procedures for measuring the time to complete the robot arm movement task, while section 6 explains the experiment results and the corresponding analysis. Finally, section 6 concludes the report with summarized findings.

\section{Related Works}

\subsection{Telerobotics system}

Telerobotic systems enable operators to interact with remote manipulators. Figure 1 shows the system in terms of the operator and the remote environments (Aracil et al., 2007). The communication channels link two environments to transmit the commands and to receive the feedback. The degree of coupling between the two environments is regarded to be weak if the operators send simple commands to the 
manipulators and then observe the outcomes. The strong coupling means that the system will rely on the operator's kinesthetic control of the remote manipulators as well as on the feedback about the ongoing operations by sensing the movements of the manipulators through the multi-modal user interface. A navigation-related command will be transformed into a series of coordinates. A symbolic command, such as pick the box up, will be translated to a corresponding sequence of remote device actions.

An accelerometer-based robot arm control system has been reported, where the human operator attached three-axis wireless accelerometers in his/her arms in order to capture the gestures and postures (Neto, Pires \& Moreira, 2009). Another example of remote robot arm control using potentiometers employed a camera mounted on the arm so as to provide visual feedback to the human operator (Aggarwal, Gaur \& Verma, 2013). There was also a robot arm control application using the artificial neural network-based image processing (AK, Topuz, \& Ersan, 2018).

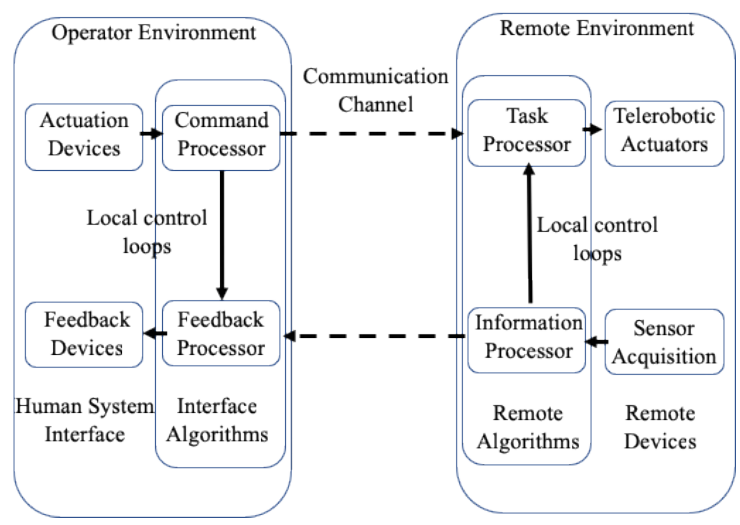

Figure 1. Telerobotic system structure

\subsection{Somatosensory Receptor}

In general, somatosensory receptors are used to capture body motion in the three-dimensional space (Xu \& Zhou, 2017). Microsoft Kinect is one such example and ASUS Xtion Pro 3D sensor bar is another one. The embedded infrared sensors capture the reflected beams from the target object and then convert them into depth information, which is in turn used to track the motion (Shafaei $\&$ Little, 2016). Specifically, both systems use the structured light approach. A sequence of known patterns is continuously projected onto an object. The cameras can observe the deformations from different directions, as some parts of the entire pattern will be distorted by the geometric shape of the object. A structured light-based measurement system extracts the depth information by comparing and analyzing the disparity from the original pattern.

\subsection{Natural User Interface}

A Natural User Interface (NUI) incorporates instinctive human behavior as a part of the human computer interaction. Some NUIs rely on the touch-free functionalities controlled by human gestures, voices, and facial expressions (Wigdor \& Wixon, 2011). Somatosensory receptors are used to enable such functionalities. For example, a Kinect-based NUI has been reported, which captured gestures and body motions to control quadrotors (Sanna et al., 2013). Another example involves the Kinect-based detection of fingertips and palm centers in order to emulate the movements of the human hands by the robotic hands (Raheja, Chaundhary \& Singal, 2011).

\section{Hardware Design and Implementation}

Figure 2 shows the components of the somatosensory sensor-based telerobotic system; Microsoft Kinect, a webcam-style add-on peripheral for video game consoles such as Xbox 360 , is a component. It enables the users to interact with the telerobotic system through a natural user interface using the gesture-based commands.

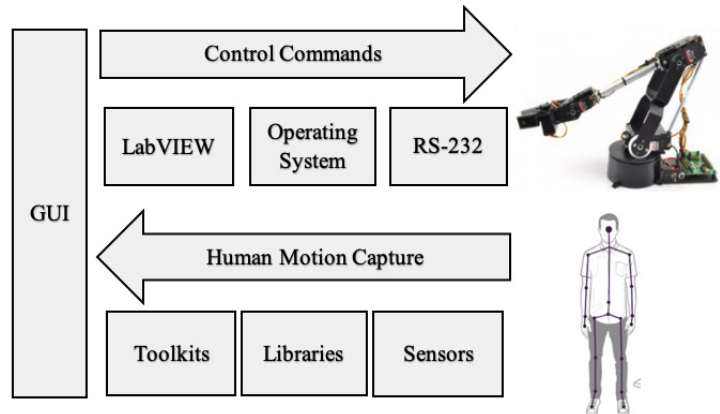

Figure 2. The components of the somatosensory sensor based telerobotic system

Kinect consists of four parts namely 1) a color sensor for capturing color images in 1280x 960 pixel resolution, 2) an infrared emitter \& depth sensor, 3) a microphone array for receiving audio signals to locate the direction of the sound source, and 4) a tilt motor which can adjust the viewing angle of the sensors. Kinect is capable of tracking the movements of 24 distinct skeletal points on the head, hands, arms, and legs (Crawford, 2017). 
Another main component of the telerobotic system is the Lynxmotion AL5D robotic arm with four Degrees of Freedom (DoF). It can hold an object weighing up to $10 \mathrm{oz}$ at full reach, and its lift capacity increases as the objects are nearer to the base of the arm (RobotShop, Inc, 2018). A camera has been installed on the shoulder of the robotic arm to provide the remote operator with a 150-degree field of front view, as shown in Figure 3. The camera is capable of recording $720 p$ resolution high-definition video.

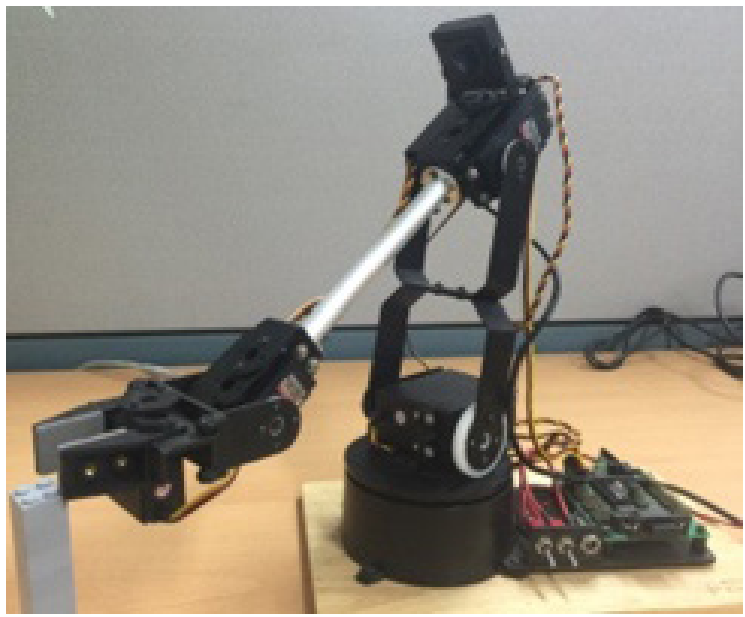

Figure 3. Camera mounted on the shoulder of the robot arm

\section{Software Design Implementation}

The Kinect API built in the LabVIEW2010 SP1 has been used to develop the software component of the telerobotic system. The software layer structure is shown in Figure 4.

\begin{tabular}{|c|c|c|c|}
\hline \multicolumn{4}{|c|}{ Graphic User Interface } \\
\hline Toolkits & & & Controls \\
\hline \multicolumn{4}{|c|}{ NI LabVIEW } \\
\hline \multicolumn{2}{|c|}{ Operating System } & \multicolumn{2}{|c|}{ Kinect Driver } \\
\hline \\
\hline
\end{tabular}

Figure 4. Telerobotic system software layer

The motion capture part consists of four Virtual Instruments (VIs), which are depicted in Figure 5 as boxes. The Initialize VI is used to create an instance of the Kinect sensor in the computer's memory. The Configure VI allows the LabVIEW developer to select from the options available for Kinect data streams, such as video and depth image resolutions. The Read VI takes the data streams from the Kinect, then processes and organizes them into a form suitable for further processing or displaying. The Close VI is used to close all of the open references created during the operation of the Kinect sensor.

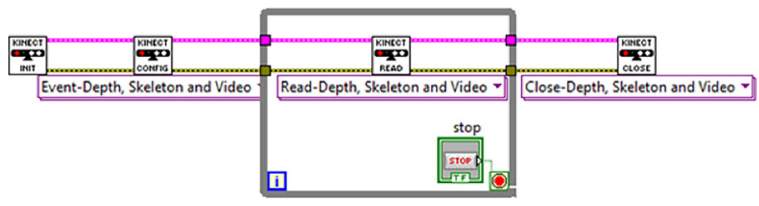

Figure 5. Kinect Virtual Instruments VIs

Although, the sensor can detect 20 joint motions, the telerobotic system only uses four out of the 12 upper limb joints, due to the limitation of the robotic arms DoF. Figure 6 shows the skeleton joints that are detectable by the Kinect (Prajapati, et al., 2018). The data regarding the displacement and distance between joints as well as the angle between joints are converted to action commands, which lead to movements of the robotic arms.

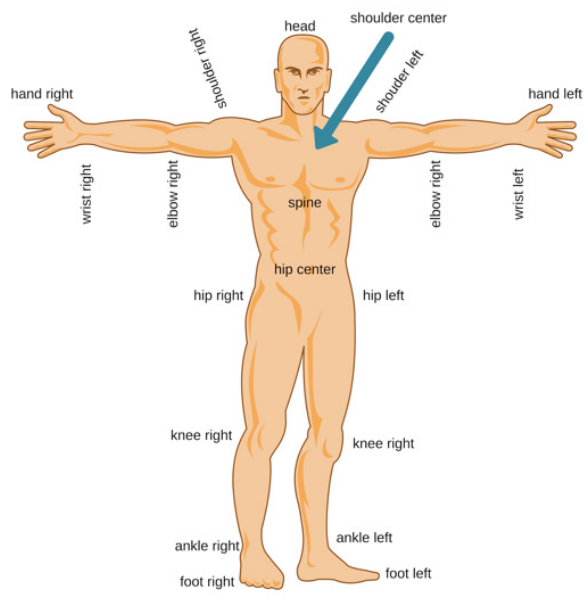

Figure 6. Skeleton joints detected by Kinect

The elbow and shoulder are used in the Kinectbased telerobotic system. The wrist is not used, as the wrist joint movement is likely to be out of the sensor detection area when the operator stretches his or her both hands horizontally. The joint angles are converted to a data stream that can be read by the servo controller. The converted data are sent via the RS-232 port to the servo controller board, then the controller drives the five servos from 0 to 180 degree.

\section{Experiments}

The primary goals of the experiments were to investigate how quickly the users of the telerobotic 
system adapt to the new interface and compare how quickly they can complete the given simple task under three experimental setups. The baseline system has allowed the subjects to move the arms of the robot by manipulating the controls on the computer screen using the mouse while directly observing the robot arm operations. The Kinectbased NUI of the telerobotic system has allowed each subject to move his/her body in order to control the robot arms while viewing the computer screen showing the robot arm operation, which has been captured by the camera attached to the arm. The other Kinect-based NUI of the telerobotic system operates in the same way, except the subjects observe the robot arm operation directly without any intervening computer screen.

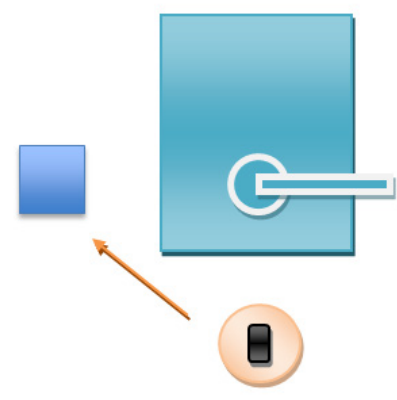

Figure 7. Experiment setting from the top

There have been 35 experimental subjects. All of them were undergraduate students, and none had any experience in operating the robot arms. The subjects have been asked to move the robot arm so as to hold a cuboid-shaped building block, move this block from the starting position to the target area as quickly as possible, then release it.

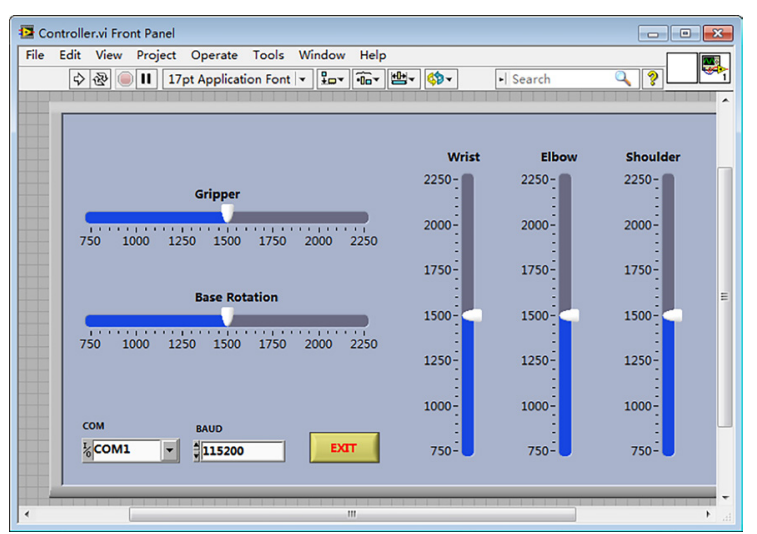

Figure 8. Mouse-based control interface

The block weighs about 40 grams. The height is 18 centimeters and the width is 2.5 centimeters. Figure 7 shows the robot arm (in the middle), the starting position (in the circle with the block on top of it), and the target area (in the square).
The arrow shows the direction from the starting position to the target area. The distance from the center of the base of the robot arm to the center of the starting position is 15 centimeters. The distance from the center of the base of the robot arm to the center of the target area is 15 centimeters. Each side of the square, which frames the target area, is 7 centimeters.

The experiments have included the automatic initialization of the robot arm's servos. The initialization time has not been included in the overall time measurement. Regarding the human subject's control of the robot arm to move the block, there has not been any constraint within the manner in which it has operated the servos.

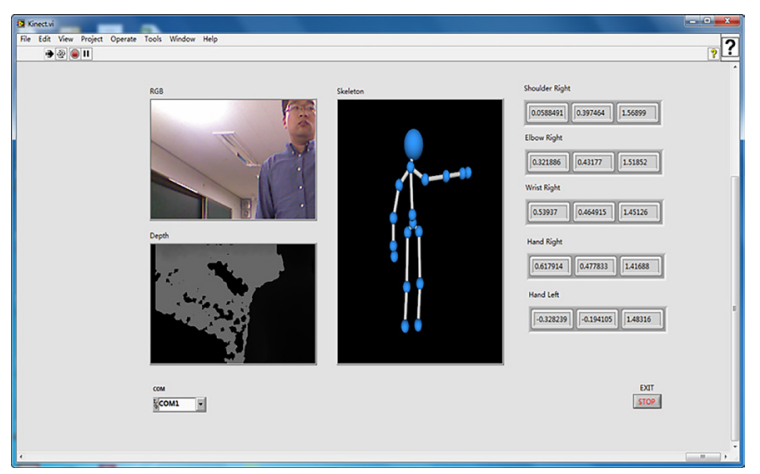

Figure 9. Gesture-based control with direct observation

The time taken to complete the task has been recorded for each trial in three settings. The mouse-based control is the baseline method where each slide bar is used to control the named servo (Figure 8).

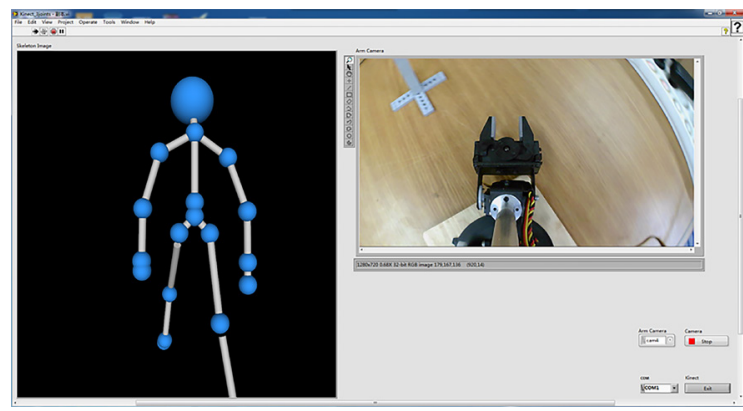

Figure 10. Gesture-based control with camera mediated observation

There are two modes of gesture-based control using the Kinect sensor. One type allows the operator to directly view the actions of the robot arms while the interface shows the RGB, depth, Isand operator's skeleton images to help to calibrate his/her standing posture (Figure 9). The other type captures the robot arm movements in the camera then displays it on the computer screen (Figure 10). 


\section{Results and Analysis}

There have been three telerobotic operation methods to investigate. Method 1, referred to as the mouse control, is the baseline mode. Method 2 involves the gesture control with the subject monitoring the robot arm's operation directly. Method 3 involves the gesture control with the subject monitoring the robot arm's operation as captured by the robot arm-mounted camera on the screen.
The subjects have had three trials for each task. The time taken to complete each trial has been recorded. There have been ten minutes of practice for each method before the experiment. The subjects have been divided into six groups. Each group has followed a different order of the object moving experiments in order to minimize the learning effects. Table 1 shows the time taken to complete the task for each method.

Table 1. Time taken to complete the task (in seconds)

\begin{tabular}{|c|c|c|c|c|c|c|c|c|c|}
\hline \multirow[t]{2}{*}{ ID } & \multicolumn{3}{|c|}{ METHOD 1} & \multicolumn{3}{|c|}{ METHOD 2} & \multicolumn{3}{|c|}{ METHOD 3} \\
\hline & Trial 1 & Trial 2 & Trial 3 & Trial 1 & Trial 2 & Trial 3 & Trial 1 & Trial 2 & Trial 3 \\
\hline 1 & 28.66 & 21.87 & 24.15 & 31.03 & 22.73 & 24.62 & 17.06 & 14.93 & 15.68 \\
\hline 2 & 25.9 & 21.68 & 23.52 & 14.98 & 12.28 & 10.76 & 20.2 & 17.21 & 14.21 \\
\hline 3 & 27.63 & 23.37 & 19.6 & 8.98 & 9.03 & 10.7 & 14.25 & 21.63 & 15.11 \\
\hline 4 & 24.62 & 18.88 & 21.33 & 18.15 & 10.2 & 14.6 & 19.13 & 19.71 & 15.25 \\
\hline 5 & 24.3 & 16.86 & 13.16 & 14.08 & 18.83 & 14.38 & 18.23 & 14.88 & 19.7 \\
\hline 6 & 19.65 & 16.76 & 18.43 & 19.48 & 14.01 & 14.58 & 15.93 & 18.54 & 13.56 \\
\hline 7 & 23.96 & 18.2 & 19.06 & 17.85 & 16.35 & 15.58 & 15.95 & 13.2 & 15.3 \\
\hline 8 & 27.38 & 28.35 & 21.36 & 16.5 & 12.63 & 14.26 & 19.83 & 13.73 & 14.18 \\
\hline 9 & 39.33 & 22.62 & 15.26 & 12.76 & 13.11 & 10.81 & 18.18 & 11.61 & 13.52 \\
\hline 10 & 36.63 & 23.05 & 18.85 & 22.75 & 42.68 & 19.82 & 41.63 & 34.88 & 36.25 \\
\hline 11 & 28.85 & 26.12 & 26.1 & 18.88 & 13.95 & 24.32 & 27.32 & 11.28 & 19.28 \\
\hline 12 & 21.7 & 14.77 & 13.53 & 26.87 & 19.53 & 18.32 & 27.03 & 52.42 & 18.65 \\
\hline 13 & 27.4 & 17.36 & 13.87 & 35.83 & 15.2 & 26.02 & 21.36 & 11.81 & 11.16 \\
\hline 14 & 18.45 & 21.35 & 21.73 & 14.38 & 14.68 & 30.05 & 17.05 & 27.67 & 16.75 \\
\hline 15 & 23.42 & 26.7 & 17.55 & 12.16 & 11.08 & 12.28 & 14.78 & 15.45 & 19.23 \\
\hline 16 & 15.81 & 15.58 & 20.15 & 10.01 & 16.2 & 13.5 & 12.18 & 19.71 & 14.78 \\
\hline 17 & 35.8 & 16.15 & 22.55 & 16.85 & 12.05 & 14.15 & 17.28 & 7.95 & 15.16 \\
\hline 18 & 14.03 & 33.01 & 15.23 & 19.58 & 11.02 & 13.55 & 10.08 & 18.41 & 26.68 \\
\hline 19 & 17.16 & 14.15 & 17.46 & 19.23 & 13.11 & 25.92 & 9.15 & 14.82 & 22.27 \\
\hline 20 & 26.57 & 20.68 & 28.47 & 41.82 & 10.5 & 24.37 & 11.51 & 14.8 & 16.51 \\
\hline 21 & 26.25 & 14.86 & 18.03 & 16.25 & 13.06 & 18.8 & 11.45 & 16.06 & 13.06 \\
\hline 22 & 28.75 & 18.09 & 12.28 & 31.86 & 19.51 & 34.72 & 12.6 & 18.15 & 13.35 \\
\hline 23 & 20.9 & 12.4 & 10.95 & 44.9 & 31.9 & 22.9 & 15.13 & 19.31 & 30.8 \\
\hline 24 & 15.18 & 18.71 & 14.06 & 16.65 & 30.2 & 20.05 & 14.68 & 14.05 & 16.23 \\
\hline 25 & 19.71 & 18.27 & 24.95 & 13.91 & 10.07 & 22.9 & 17.03 & 17.72 & 21.13 \\
\hline 26 & 28.53 & 19.35 & 15.78 & 9.2 & 11.93 & 38.8 & 15.71 & 26.7 & 16.2 \\
\hline 27 & 35.75 & 20.58 & 17.07 & 10.25 & 21.43 & 12.56 & 12.25 & 33.88 & 14.46 \\
\hline 28 & 38.87 & 32.62 & 27.02 & 22.3 & 15.58 & 25.6 & 19.22 & 11.1 & 21.88 \\
\hline 29 & 27.75 & 20.43 & 20.38 & 14.41 & 16.95 & 18.82 & 15.16 & 31.95 & 21.06 \\
\hline 30 & 26.45 & 20.32 & 16.68 & 33.98 & 13.48 & 13.91 & 15.55 & 13.72 & 13.18 \\
\hline 31 & 30.48 & 29.25 & 28.8 & 18.48 & 19.3 & 22.92 & 16.43 & 11.36 & 18.55 \\
\hline 32 & 17.21 & 10.55 & 10.5 & 16.56 & 15.38 & 40.83 & 10.25 & 10.03 & 12.79 \\
\hline 33 & 19.47 & 59.9 & 13.81 & 46.13 & 44.8 & 86.2 & 15.43 & 24.55 & 32.06 \\
\hline 34 & 24.08 & 18.17 & 14.81 & 22.72 & 10.51 & 17.85 & 13.83 & 9.15 & 24.87 \\
\hline 35 & 21.2 & 15.16 & 17.58 & 27.05 & 16.1 & 16.94 & 14.98 & 16.56 & 16.08 \\
\hline
\end{tabular}




\subsection{Task Completion Time by Methods}

The central hypothesis was that it would be quicker to operate the robot arm with the gesturebased control than the mouse-based one. The task completion times have been compared in order to verify the hypothesis.

Thirty-five subjects have undergone three trials for each method, and thus there have been 105 trials for each method. Table 2 shows that the gesture-based control while monitoring via the camera (i.e., method 3) has taken the shortest time for subjects to complete the task. The gesture-based control while directly monitoring the operation (i.e., method 2) has taken more time than the gesture-based control while monitoring via camera, but less time than the mouse-based control (i.e. method 1).

Table 2. Descriptive statistics about the three methods

\begin{tabular}{|c|c|c|c|}
\hline & Mean & Std. Deviation & N \\
\hline Method 1 & 21.7910 & 7.30703 & 105 \\
\hline Method 2 & 20.0246 & 10.93414 & 105 \\
\hline Method 3 & 17.8637 & 7.02861 & 105 \\
\hline
\end{tabular}

As the same subjects have tried all three methods, one-way repeated measures ANOVA has been used to analyze the data. Mauchly's test of sphericity has shown that the assumption of sphericity has not been met, $\mathrm{X} 2(2)=10.275, \mathrm{p}=.006$. A repeated measures ANOVA with a Greenhouse-Geisser correction has determined a statistically significant difference between the methods $(\mathrm{F}(1.827,189.964)$ $=6.123, \mathrm{P}<0.005)$ in mean task completion time.

Table 3. Pairwise comparisons of time by methods

\begin{tabular}{|c|c|c|c|c|c|c|}
\hline \multicolumn{5}{|c|}{ Measure: time } & \multicolumn{2}{|c|}{$\begin{array}{l}95 \% \text { Confidence } \\
\text { Interval for } \\
\text { Difference }^{b}\end{array}$} \\
\hline $\begin{array}{l}\text { (I) } \\
\text { method }\end{array}$ & $\begin{array}{l}(\mathrm{J}) \\
\text { method }\end{array}$ & $\begin{array}{c}\text { Mean } \\
\text { Difference } \\
(\mathrm{I}-\mathrm{J})\end{array}$ & $\begin{array}{l}\text { Std. } \\
\text { Error }\end{array}$ & Sig. ${ }^{b}$ & $\begin{array}{l}\text { Lower } \\
\text { Bound }\end{array}$ & $\begin{array}{l}\text { Upper } \\
\text { Bound }\end{array}$ \\
\hline \multirow{2}{*}{1} & 2 & 1.766 & 1.262 & .494 & -1.304 & 4.937 \\
\hline & 3 & $3.927^{*}$ & .958 & .000 & 1.595 & 6.259 \\
\hline \multirow{2}{*}{2} & 1 & -1.766 & 1.262 & .494 & -4.837 & 1.304 \\
\hline & 3 & 2.161 & 1.132 & .177 & -.593 & 4.914 \\
\hline \multirow{2}{*}{3} & 1 & $-3.927^{*}$ & .958 & .000 & -6.259 & -1.595 \\
\hline & 2 & -2.161 & 1.132 & .177 & -4.914 & .593 \\
\hline \multicolumn{7}{|c|}{ Based on estimated marginal means } \\
\hline b. Adju & $t$ fo & iple con & on & nt & & \\
\hline
\end{tabular}

Table 3 shows that the post hoc tests using the Bonferroni correction have revealed that changing the telerobotic control method has elicited a slight reduction in the task completion time from method 1 to method $2(21.79 \pm 7.31 \mathrm{sec}$ vs 20.02 $\pm 10.93 \mathrm{sec}$, respectively), but this has not been statistically significant $(\mathrm{p}=.494)$. However, the task completion time has been reduced to $17.86 \pm$ $7.03 \mathrm{sec}$ for method 3, which has been statistically significantly different from that of method 1 ( $p<$ $.005)$. Therefore, it can be concluded that the use of gesture-based control while monitoring via camera elicits a statistically significant reduction in the task completion time from the mouse-based control, but not from the gesture-based control while directly monitoring the operation.

\subsection{Task Completion Time by Trials}

Each subject has undergone three trials for each method. There has not been any restriction regarding the duration of the rest on period between the trials, but no subject has taken more than five minutes rest. Another hypothesis is that the task completion time would be shortened more for the mouse-based control and less for the gesture-based control as the trial progresses.

Table 4. Descriptive statistics about the three trials of the mouse-based control method

\begin{tabular}{|c|c|c|c|}
\hline & Mean & Std. Deviation & $\mathrm{N}$ \\
\hline Trial 1 & 25.3666 & 6.59493 & 35 \\
\hline Trial 2 & 21.3191 & 8.52462 & 35 \\
\hline Trial 3 & 18.6874 & 4.88376 & 35 \\
\hline
\end{tabular}

Thirty-five subjects have undergone three trials for the mouse-based control method. Table 4 shows that the third trial has taken the shortest time for the subjects to complete the task. The second trial has taken more time than the third trial but less time than the first trial.

As the same subjects have tried the mouse-based control method three times, one-way repeated measures ANOVA has been used to analyze the data. Mauchly's test of sphericity has shown that the assumption of sphericity has not been met, $\mathrm{X} 2(2)=7.24, p=.027$. A repeated measures ANOVA with a Greenhouse-Geisser correction has determined a statistically significant difference between the trials $(\mathrm{F}(1.671,56.813)=10.153, \mathrm{P}<$ $.005)$ in mean task completion time. 
Post hoc tests using Bonferroni correction have revealed that conducting more trials has elicited a slight reduction in the task completion time from the first to the second trial $(25.37 \pm 6.59 \mathrm{sec}$ vs. $21.32 \pm 8.52 \mathrm{sec}$, respectively), which has not been statistically significant $(\mathrm{p}=.078)$. However, the task completion time for the third trial has been reduced to $18.69 \pm 4.88 \mathrm{sec}$, which has been statistically significantly different from that of the first trial $(\mathrm{p}<.005)$. Therefore, it can be concluded that the third trial elicits a statistically significant reduction in the task completion time from the first trial, but not from the second trial.

Thirty-five subjects have also undergone three trials for the gesture-based control while directly monitoring the operation method. Table 5 shows that the second trial has taken the shortest time for subjects to complete the task. The first trial has taken more time than the second trial but less time than the third trial.

Table 5. Descriptive statistics about the three trials of the gesture-based control while directly monitoring the operation method

\begin{tabular}{|c|c|c|c|}
\hline & Mean & Std. Deviation & $\mathrm{N}$ \\
\hline Trial 1 & 21.0520 & 9.91819 & 35 \\
\hline Trial 2 & 17.1249 & 8.39802 & 35 \\
\hline Trial 3 & 21.8969 & 13.54441 & 35 \\
\hline
\end{tabular}

As the same subjects have tried the gesture-based control while directly monitoring the operation method three times, one-way repeated measures ANOVA has also been used to analyze the data. Mauchly's test of sphericity has shown that the assumption of sphericity has been met, X2(2) = $2.772, p=.250$. A repeated measures ANOVA with sphericity assumption has determined a statistically significant difference between the trials $(\mathrm{F}(2,68)=3.592, \mathrm{P}<.05)$ in mean task completion times.

Post hoc tests using Bonferroni correction have revealed that conducting more trials has elicited a reduction in the task completion time from the first to the second trial $(21.05 \pm 9.92$ sec vs. $17.12 \pm 8.40 \mathrm{sec}$, respectively), which has not been statistically significant $(\mathrm{p}=.063)$. However, the task completion time for the third trial has been slightly increased to 21.90 $\pm 13.54 \mathrm{sec}$, which has not been statistically significantly different from that of the first trial $(\mathrm{p}=1.000)$. The task completion time has not been statistically significantly increased from the second to the third trial $(\mathrm{p}=.061)$. Therefore, it can be concluded that no trial elicits any statistically significant reduction or increase in the task completion time, although the overall mean task completion times have been found to be statistically significantly different.

Table 6. Descriptive statistics about the three trials of the gesture-based control while monitoring via the camera method

\begin{tabular}{|c|c|c|c|}
\hline & Mean & Std. Deviation & $\mathrm{N}$ \\
\hline Trial 1 & 16.7951 & 5.91818 & 35 \\
\hline Trial 2 & 18.5409 & 8.95435 & 35 \\
\hline Trial 3 & 18.2551 & 5.83948 & 35 \\
\hline
\end{tabular}

Thirty-five subjects has undergone three trials for the gesture-based control while monitoring via the camera method. Table 6 shows that the first trial has taken the shortest time for subjects to complete the task. The third trial has taken more time than the first trial but less time than the second trial.

As the same subjects have tried the gesture-based control while monitoring via the camera method three times, one-way repeated measures ANOVA has been used to analyze the data. Mauchly's test of sphericity has shown that the assumption of sphericity has been met, X2(2) $=4.471, \mathrm{p}=.107$. A repeated measures ANOVA with sphericity assumption has been determined a statistically significant between the trials $(\mathrm{F}(2,68)=.882, \mathrm{P}=$ .419) in mean task completion times. Therefore, it can be concluded that no trial elicits any statistically significantly reduction or increase in the task completion time.

\section{Conclusion}

A Kinect-based telerobotic system has been developed to test the feasibility of gesture-based control of remote manipulators such as robot arms. The gesture-based system has been compared with the baseline mouse-based control system in terms of the task completion time.

There have been two variations in the gesturebased control. One type has allowed the subjects to monitor the robot arm operation directly while the other type has restricted the subjects' observation to the robot arm movements captured by the robot arm-mounted camera. The movements have 
then been shown on a screen in real-time. The second method is a realistic telerobotic system in comparison to the first method. However, both methods have been included in the experiments in order to understand the effects of indirect observation using the camera.

The primary hypothesis of the present work was that the gesture-based control would enable the test subjects to complete the given task in a shorter time than the mouse-based control. The hypothesis was proven to be correct for the gesture-based control while monitoring via the camera but not for the gesture-based control while directly watching the robot arm movements. The secondary hypothesis was that the task completion time would be shortened as the subjects practiced to a greater extent. This hypothesis was also found

\section{REFERENCES}

1. AK, A., Topuz, V. \& Ersan, E. (2018). Visual Servoing Application for Inverse Kinematics of Robotic Arm Using Artificial Neural Networks, Studies in Informatics and Control, 27(2), 183-190. DOI: doi. org/10.24846/v27i2y201806

2. Aggarwal, L., Gaur, V. \& Verma, P. (2013). Design and Implementation of a Wireless Gesture Controlled Robotic Arm with Vision, International Journal of Computer Applications, 79(13), 39-43.

3. Aracil, R., Buss, M., Cobos, S., Ferre, M., Hirche, S., Kuschel, M. \& Peer, A. (2007). The Human Role in Telerobotics, Advances in Telerobotics (editors: Ferre, M., Buss, M., Aracil, R., Melchiorri, C. \& Balaguer, C.), 11-24.

4. Crawford, S. (2017). How Microsoft Kinect Works, HowStuffWorks. Available at: <https://electronics.howstuffworks.com/ microsoft-kinect2.htm $>$, last accessed: Sept. $15,2017$.

5. Neto, R., Pires, N. \& Moreira, P. (2009). Accelerometer-Based Control of an Industrial Robotic Arm, International Journal of Electronics, 6, 167-173.

6. Prajapati, A., Bhatt, M., Joshi, Y. \& Negi, R. (2018). Skeleton Tracking Using Microsoft Kinect for Windows on Matlab, International Journal of Recent Research Aspects, 5(1), 377-380. to be true for the mouse-based control, but not for both types of gesture-based control.

These findings lead to the following conclusions. The camera-based monitoring and gesture-based natural user interface was the best method for controlling the telerobotic system. Such an interface was also the easiest to learn to use, as there were no task completion time differences between the trials.

One of the practical goals of this paper was to develop a telerobotic system that is easy to learn and use, even for the seniors, by employing a gesture-based natural user interface so that they can work on physically challenging tasks in work areas such as agriculture, farming or mining. The outcome of this research is believed to be a positive step toward achieving this goal.

7. Raheja, J., Chaundhary, A. \& Singal, K. (2011). Tracking of fingertips and centers of palm using Kinect. In Proceedings of the 2011 Third International Conference on Computational Intelligence, Modelling \& Simulation (pp. 248-252).

8. RoboShop, Inc. (2018). AL5D. Available at: $<$ http://www.lynxmotion.com/c-130-al5d. aspx $>$, last accessed: Feb. 23, 2018.

9. Sanna, A., Lamberti, F., Paravati, G., Ramirez, E. \& Manuri, F. (2013). A Kinectbased natural interface for quadrotor control, Entertainment Computing, 4(3), 179-186.

10. Shafaei, A. \& Little, J. (2016). Real-Time Human Motion Capture with Multiple Depth Cameras. In Proceedings of the 13th Conference on Computer and Robot Vision (pp. 24-31).

11. Wigdor, D. \& Wixon, D. (2011). Brave NUI world: designing natural user interfaces for touch and gesture. Elsevier.

12. Xu, T. \& Zhou, Y. (2017). Fall Detection Based on Skeleton Data, Human Aspects of IT for the Aged Population Applications, Services and Contexts (editors: Zhou Y. \& Salvendy, G.), 475-486. 